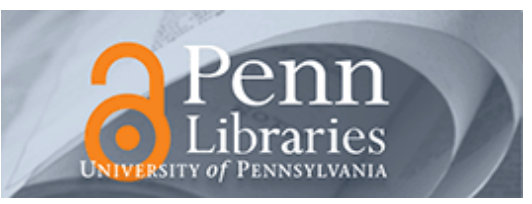

University of Pennsylvania

ScholarlyCommons

\title{
Robot Assembly: Another Source of Nonholonomic Control Problems
}

\author{
Daniel E. Koditschek \\ University of Pennsylvania, kod@seas.upenn.edu
}

Follow this and additional works at: https://repository.upenn.edu/ese_papers

Part of the Electrical and Computer Engineering Commons, and the Systems Engineering Commons

\section{Recommended Citation}

Daniel E. Koditschek, "Robot Assembly: Another Source of Nonholonomic Control Problems", , 1627-1632. March 1991.

Copyright 1991 IEEE. Reprinted from Proceedings of the American Control Conference March 1991, pages 1627-1632.

This material is posted here with permission of the IEEE. Such permission of the IEEE does not in any way imply IEEE endorsement of any of the University of Pennsylvania's products or services. Internal or personal use of this material is permitted. However, permission to reprint/republish this material for advertising or promotional purposes or for creating new collective works for resale or redistribution must be obtained from the IEEE by writing to pubs-permissions@ieee.org. By choosing to view this document, you agree to all provisions of the copyright laws protecting it.

NOTE: At the time of publication, author Daniel Koditschek was affiliated with Yale University. Currently, he is a faculty member in the Department of Electrical and Systems Engineering at the University of Pennsylvania.

This paper is posted at ScholarlyCommons. https://repository.upenn.edu/ese_papers/682

For more information, please contact repository@pobox.upenn.edu. 


\title{
Robot Assembly: Another Source of Nonholonomic Control Problems
}

\author{
Abstract \\ Assembly problems require that a robot with a few actuated degrees of freedom manipulate an \\ environment with a greater number of unactuated degrees of freedom. Since the dynamical coupling \\ between degrees of freedom in this setting is a function of their relative configuration, the motion of such \\ systems is subject to constraints that preclude smooth feedback stabilization. This paper explores the \\ extent to which assembly planning and control may be effected by recourse to some other methodical \\ means of generating stabilizing feedback controllers. A partial solution is offered for a very simple \\ assembly problem involving an intermittent dynamical environment. \\ For more information: Kod*Lab \\ Disciplines \\ Electrical and Computer Engineering | Engineering | Systems Engineering \\ Comments \\ Copyright 1991 IEEE. Reprinted from Proceedings of the American Control Conference March 1991 , \\ pages 1627-1632. \\ This material is posted here with permission of the IEEE. Such permission of the IEEE does not in any way \\ imply IEEE endorsement of any of the University of Pennsylvania's products or services. Internal or \\ personal use of this material is permitted. However, permission to reprint/republish this material for \\ advertising or promotional purposes or for creating new collective works for resale or redistribution must \\ be obtained from the IEEE by writing to pubs-permissions@ieee.org. By choosing to view this document, \\ you agree to all provisions of the copyright laws protecting it. \\ NOTE: At the time of publication, author Daniel Koditschek was affiliated with \\ Yale University. Currently, he is a faculty member in the Department of Electrical \\ and Systems Engineering at the University of Pennsylvania.
}




\title{
Robot Assembly: Another Source of Nonholonomic Control Problems
}

\author{
Preprint of a paper to appear in the \\ 1991 American Control Conference \\ Boston, MA \\ Daniel E. Koditschek * \\ Center for Systems Science \\ Yale University, Department of Electrical Engineering
}

March 8, 1991

\begin{abstract}
Assembly problems require that a robot with a few actuated degrees of freedom manipulate an environment with a greater number of unactuated degrees of freedom. Since the dynamical coupling between degrees of freedom in this setting is a function of their relative configuration, the motion of such systems is subject to constraints that preclude smooth feedback stabilization. This paper explores the extent to which assembly planning and control may be effected by recourse to some other methodical means of generating stabilizing feedback controllers. A partial solution is offered for a very simple assembly problem involving an intermittent dynamical environment.
\end{abstract}

\section{Introduction}

In the course of our robot juggling research [5] we have recently reported experimental success in transferring a two degree of freedom body falling freely in the earth's gravitational field from an arbitrarily chosen initial state to a specified zero velocity state via intermittent impacts with a one degree of freedom robot [4]. Our control strategy commands first a (provably correct) juggling algorithm [3] designed to bring the body to some known periodic trajectory and then switches to a catching algorithm designed (but not yet formally proven) to bring the body to rest from the known intermediate oscillatory conditions. Empirical success notwithstanding, one would prefer, in general, some methodically chosen controller. One might inquire, for instance, why this apparently ad hoc strategy should be employed, and

"This work was supported in part by the National Science Foundation under a Presidential Young Investigator Award. whether other intuitively devised schemes might succeed equally well. In particular, it seems undesirable to rely upon a switched combination of two feedback laws when a single smooth controller might do the job.

Transferring a system of bodies possessing a greater number of degrees of freedom than the number of actuated degrees of freedom available from one configuration to another is an assembly problem. Note that the possibility of solving such problems depends on the fact that the dynamical coupling between degrees of freedom is not fixed. More precisely, the allowable infinitesimal motions of the body system differ from configuration to configuration. Thus, since the motion of the bodies is subject to constraints that are not integrable, assembly problems give rise to nonholonomically constrained control systems. These seem to differ from the more commonly studied nonholonomic robotics problems involving steering wheels [11] or coupled vehicles [9] in that here the tangent bundle constraints are (at best) $C^{\infty}$ rather than analytic. They seem to be very similar in most other respects, and there is good reason to hope that a principled treatment of one class should shed light on the other. One significant feature of such problems is that even while the control systems that arise are completely controllable, Brockett's [2] stabilizability condition is not met [1], and there is no possibility of constructing a single smooth feedback controller to stabilize an isolated equilibrium state.

These preliminary remarks now place the juggling problem in a clearer context. While our empirically successful "materials handling" strategy has still little pretension to systematic origins, it is at least clear that some kind of "switched" control policy will be unavoidable. In a recent paper introducing the assembly problem [8], the author has studied a very simple ex- 
ample - the One Degree of Freedom Unit Asscmbly Problem - in a quasi-static environment. That is to say, the Newtonian dynamics of a free body are replaced with Whitney's "generalized damper" $d y$ namics [12] as is common in the assembly planning literature [10], the system is shown to be completely controllable and not stabilizable, and a switched controller derived (relatively) methodically is shown to solve a partial version of the problem.

This paper represents a first effort to come to grips (so to speak) with the possibility for justification (or improvement) of our experimental success in "materials handling" through juggling and catching via a formal examination of the attendant assembly problem. Section 2 places the One Degree of Freedom Unit Assembly Problem back in a dynamical setting - the free body obeys Newton's laws - and demonstrates that any physically reasonable model of contact between the robot and body gives rise to a dynamical model which is again completely controllable but not feedback stabilizable. To simplify the synthesis problem, it is convenient to pass to an impulse model of contact, thereby fixing the formal representation of the problem as occuring within an intermittent dynamical environment in conformity with our ongoing juggling work [5]. A partial solution to this assembly problem is offered in Section 3. The paper concludes with a brief speculation about where these ideas may lead.

\section{The One Degree of Freedom Unit Assembly Problem}

Consider a point unit mass body restricted to a single horizontal axis of motion, $b \in \mathbb{R}$, governed, in isolation, by Newton's law, $\ddot{b}=0$. Consider as well a point unit mass robot restricted to a single axis of motion parallel ${ }^{1}$ to that of the body, $r \in \mathbb{R}$. The robot is controlled by a force we are free to command, $\phi$, and its motion is governed by Newton's laws, $\ddot{r}=\phi$. Let the robot have a position controlled "gripper", $\operatorname{GRIP}(t)$, which, when extended, ( GRIP $=1$ ) is capable of engaging the body when the two point masses are aligned at the same relative positions on their respective axes.

\footnotetext{
${ }^{1}$ It would be more appropriate to place the robot and body in the same configuration space. This would merely complicate the gripbper model in the present circumstances since a model of pulling would necessarily be required in addition to the simple model of pushing - impacts - introduced below. In higher degree of freedom problems this complication will not arise and the robot and bodies may be located in the same space.
}

\subsection{The Task}

It is desired to place the body at a specified goal location. The problem at hand is to devise an autonomous strategy for the robot that will enable it. to move toward the body, "grab it", and place it in the arbitrarily designated new location, and proceed to its nest. More formally, we seek a feedback controller, $(\phi$, GRIP $)(t)=G(r, \dot{r}, b, \dot{b}, \ldots)(t)$ which causes the robot to bring the body to the specified goal assuming arbitrary initial conditions. There are two versions of the problem. In the first instance, the robot's state is left unspecified after the body has reached the goal. In the second instance, the robot is commanded to return to some specified nest state as well. This paper provides a smooth feedback controller, $G$ that solves the first version -.. "half" — of the unit assembly problem. It will be seen that the second version cannot be effected via a smooth feedback controller because of the intrinsic nonholonomic constraints that arise. This is the problem solved by empirical demonstration in [4].

\subsection{Contact Models}

This section addresses the issue of how to represent the contact event between body and robot gripper. Dynamical assembly interactions comnote in their simplest instance contact conditions between body and robot that are of extremely short duration and involve very large forces. In the limit, such impact conditions lead to the notion of impulses introduced to maintain inequality constraints in configuration [7]. In the present setting it will prove useful to work with both the continuous and impulsive models of contact force. To maintain some generality in the later controllability/stabilizability discussion a general force model of contact is first presented that makes the minimal assumptions necessary to realize the required physical constraints. For purposes of controller synthesis it proves convenient to replace this general force law with an impulse model of instantaneous collision.

\subsubsection{Force Model}

Contact forces, $c(\delta, \dot{\delta})$, depend upon the relative configuration, $\delta=b-r$. Roughly speaking, they obtain when the body and robot's gripper approac each other in close proximity. That is, given some small "contact region", $\epsilon>0$, we will require

$$
c(\delta, v) \begin{cases}>0 & 0<\delta<\epsilon, v<0 \\ <0 & -\epsilon<\delta<0, v>0 \\ =0 & \text { otherwise }\end{cases}
$$

according to the arguments of $[7, \S 2.3$. B.ii.c]. Clearly such a function cannot be analytic, but it is expedi- 
ent to assume smoothness in order to avoid techmical issues of existence and uniqueness in the sequel. ${ }^{2}$

As matters stand, this crude model does not prohibit the possibility that sufficiently large and opposing velocities might give rise to trajectories whose relative configuration changes sign. Such trajectories would violate the physical principle that two bodies camnot occupy the same space at the same time. This principle may be enforced by letting $c$ become unbounded as $\delta \rightarrow 0$, or, effectively the same, assuming that the initial kinetic energy of the joint system is limited (and that $c$ does not add energy to the system) as a function of the maximal value of $c$.

\subsubsection{Impulse Model}

In the limit, as $|c| \rightarrow \infty$, the time over which the contact forces are engaged becomes vanishingly small, and the integral of work done remains a finite value called the "impulse" $[7, \S 2.5]$. Energy is generally lost (the contact forces are not conservative) during the impact, and this leads to the notion of a coefficient of restitution $[7, \S 4.2]$,

$$
\dot{b}_{F}-\dot{r}_{F}=-\alpha\left(\dot{b}_{I}-\dot{r}_{I}\right) \text {, }
$$

where the subscripts $I, F$, denote initial and final velocities respectively. Generally speaking, one knows the initial velocities and desires to predict the final velocities. To do so, introduce one last assumption, $\dot{r}_{I}=\dot{r}_{F}$, indicating the ability of the robot's controlled source of external forces to effectively cancel out any impact effects. This implies

$$
\dot{b}_{F}=-\alpha \dot{b}_{I}+(1+\alpha) \dot{r} \text {. }
$$

\subsection{The Control Problem}

This section addresses the sytems theoretic properties of the coupled robot-body system that arise from the models introduced above. The general force model is used to show that while this system is completely controllable, it is not stabilizable - that is, no isolated equilibrium state may be made asymptotically stable via a smooth feedback law, $G$. The impulse model is then used to derive an abstracted representation of the robot-body interaction that we have previously termed the Environmental Control Problem [5].

\subsubsection{Qualitative Properties of the Force Model}

The robot is controlled by a perfect (but bounded) force, $\phi$. Dynamical coupling occurs through contact

\footnotetext{
${ }^{2}$ The function cannot even be differentiable at the origin, but it will prove necessary to avoid this regime anyway through the methods below.
}

forces (1) arising from the robot's gripper engaging the body: if the robot exerts a force $c$ on the body then the body exerts a force, $-c$, on the robot. But this contact occurs only if the robot's gripper is engaged. The simplest representation of this situation may be given by multiplying the force law, $c$, by the multiplicative decision variable, GRIP, which, when zero, precludes any dynamical coupling at all. The coupled system may be modeled as

$$
\begin{gathered}
\ddot{r}=-\operatorname{GRIP} \cdot c+\phi \\
\ddot{b}=\mathrm{GRIP} \cdot c .
\end{gathered}
$$

Denoting the joint state vector as $x=[b, \dot{b}, r, \dot{r}]^{\mathrm{T}}$, this dynamical system may be rewritten as

$$
\dot{x}=\left[\begin{array}{c}
x_{2} \\
-\operatorname{GRIP} \cdot c\left(x_{1}-x_{3}, x_{2}-x_{4}\right) \\
x_{4} \\
\operatorname{GRIP} \cdot c\left(x_{1}-x_{3}, x_{2}-x_{4}\right)+\phi
\end{array}\right]
$$

It is easy to see that this system is completely controllable. Given initial and final states, $x_{I}, x_{F}$, respectively, set GRIP $=0$, and transfer the decoupled robot (double integrator) system to match the state of the body at some future time, $t_{1}$, where matching means bringing the robot to the boundary of the $\epsilon$ region of contact at a matching velocity so that the robot and the body's goal point form an interval containing the body's present location, $x_{I 1}+t_{1} x_{12} \in\left[x_{F_{1}}, x_{3}\left(l_{1}\right)\right]$. Now engage the gripper (set GRIP $=1$ ), cancel the effects of contact on the robot (set $\phi=-c+\hat{\phi}$ ), and drive the body's state until time $t_{2}$ in such a manner that it will "drift" to the desired final state, $\left(x_{F 1}, x_{F_{2}}\right)$ at time $t_{3}$. This may be done by using $\hat{\phi}$ to control the relative position and velocity of the robot and body, thereby employing $c$ as a control input to the body. Disengage the gripper at time $t_{2}$ and bring the robot to its desired final state at $t_{3}$ via the input $\phi$.

Lemma 1 The coupled system (3) is not smoothly stabilizable. to any isolated equilibrium state, $x^{*}$ for which $\left|x_{1}^{*}-x_{3}^{*}\right|>\epsilon$.

Proof: According to Brockett's observation [2], the vector field must be onto some neighborhood of the origin. But $c \equiv 0$ in a neighborhood every hypothesized $x^{*}$, thus the second component of the vector field is always zero on that neighborhood, and it camnot be onto any open ball about 0 .

\subsubsection{Impulse Model}

Letting $\dot{r}(n)$ denote the velocity of the robot at impact $n$ and $\Delta t_{n} \triangleq t_{n+1}-t_{n}$ denote the time the robot 
allows to elapse between impact $n$ and impact $n+1$, we have

$$
\begin{aligned}
& b(n+1)=b(n)+\Delta t_{n}(-\alpha \dot{b}(n)+(1+\alpha) \dot{r}(n)) \\
& \dot{b}(n+1)=-\alpha \dot{b}(n)+(1+\alpha) \dot{r}(n) .
\end{aligned}
$$

While both $\Delta t_{n}$ and $i(n)$ are formally control inputs, it will suffice for the present purposes to concentrate on the second. Thus, denoting

$$
w(n)=\left[\begin{array}{c}
b\left(t_{n}\right) \\
\dot{b}\left(t_{n}\right)
\end{array}\right] ; \quad u(n)=\dot{r}\left(t_{n}\right)-\dot{b}\left(t_{n}\right),
$$

the system may be equivalently rewritten in the form

$$
\begin{aligned}
w(n+1) & =f(w(n), u(n)) \\
f(w, u) & =A w+h u \\
A & =\left[\begin{array}{cc}
1 & \Delta t_{n} \\
0 & 1
\end{array}\right] \\
h & =\left[\begin{array}{c}
\Delta t_{n} \\
1
\end{array}\right](1+\alpha) \text { GRIP. }
\end{aligned}
$$

This system is analogous to what we have termed the environmental control system in our juggling work [5]. It's dynamics express all the logically possible outcomes of robot-body collisions abstracting away any consideration of the robot's intrinsic dynamics.

We now seek an "impact schedule" - that is, a function, $g: \mathcal{W} \rightarrow \mathcal{U}$ such that the closed loop dynamics $f_{g}(w)=f(w, g(w))$ has a globally asymptotically stable goal state, $w^{*}$. Assuming a constant choice of $t_{n}$, this problem is readily solved via an appeal to linear systems theory. For example, it is easy to see that

$$
w^{*}=A w^{*}+h u
$$

has a solution, $u$, if and only if $w_{2}^{*}=0$. Thus, not surprisingly, only zero velocity goal points may be made asymptotically stable fixed points by an appropriate choice of $g$. Notice that $f$ is invariant under all zerovelocity translations, that is, if $T(w) \triangleq w-\left(w_{1}^{*}, 0\right)$ then $T \circ f\left(T^{-1}(w), u\right)=f(w, u)$, so there is no loss of generality in assuming $w_{1}^{*}=0$ as well. Finally, since this system is completely controllable,

$$
\operatorname{Det}[h, A h]=(1+\alpha)^{2} t_{n},
$$

it is now clear that any fixed point stabilization problem may be solved by an appropriate choice of gains,

$$
g(w)=k^{\mathbf{T}} w ; \quad k=\left[\begin{array}{c}
k_{1} \\
k_{2}
\end{array}\right] .
$$

\section{Solving the Partial Assem- bly Problem}

In our juggling problem [5] the environmental control system (4) was nonlinear in state as well as input, and linearized feedback strategies produced closed loop systems, $f_{g}$ with unacceptably small domains of attraction [3]. It was necessary to pass to a nonlinear strategy. In the present case, there can be no objection to a linear feedback law since an asymptotically stable equilibrium state attracts the entire state space. However the difficulties in implementing the abstract impact schedule - in measuring the body state just at time of impact; in controlling the robot's velocity acurately enough at the exactly right time and place required - and the absolute reliance upon the abstract dynamical model implied by the map $g$ turned out to degrade juggling performance considerably.

In contrast, extremely reliable juggling behavior with very strong robustness properties emerged from forcing the robot to track a reference trajectory obtained by passing the body's state trajectory through a carefully chosen memoryless nonlinearity $[5,3]$. Thus, in setting up the present problem in Section 2, we have explicitly required that the robot's feedback controller, $G$ be a function of the continuous time robot-body state.

\subsection{Augmented Reference Dynamics}

Forcing the robot to track the output of a memoryless nonlinear transformation of the body's state,

$$
r(t)=\mu(b, \dot{b})(t)
$$

achieved juggling in an intermittent dynamical environment forced by the earth's gravitational field [5]. In this setting, the same strategy will not work. For example, if $\dot{b}(0)=0$ but $b \neq 0$ then $\dot{r}(0)=0$, and the first (and all succeeding) impacts to the environmental control system (4) are zero, and no motion occurs.

This problem will be circumvented by adding memory to the controller. Consider the second order dynamical system

$$
\dot{a}=\left[\begin{array}{cc}
0 & 1 \\
-\omega^{2}\left(1+\beta^{2}\right) & -2 \omega \beta
\end{array}\right] a+\left[\begin{array}{l}
0 \\
1
\end{array}\right] d
$$

where both $\omega, \beta$ are positive so that the system has complex conjugate poles and oscillatory solutions obtain. Forcing this system with the output of the body,

$$
d \triangleq\left[\omega\left(1+\beta^{2}\right), 2 \omega \beta\right]\left[\begin{array}{l}
b \\
\dot{b}
\end{array}\right]
$$

and defining error coordinates as $\left(e_{1}, e_{2}\right)=(b, \dot{b})-$ $\left(a_{1}, a_{2}\right)$ implies

$$
\dot{e}=\left[\begin{array}{cc}
0 & 1 \\
-\omega^{2}\left(1+\beta^{2}\right) & -2 \omega \beta
\end{array}\right] e .
$$


It is now convenient to introduce a change of basis,

$$
W \triangleq\left[\begin{array}{cc}
\omega \sqrt{1+\beta^{2}} & \frac{\beta}{\sqrt{1+\beta^{2}}} \\
0 & \frac{1}{\sqrt{1+\beta^{2}}}
\end{array}\right],
$$

to the complex normal form [6],

$$
\bar{A} \triangleq W A W^{-1}=\omega\left[\begin{array}{cc}
-\beta & 1 \\
-1 & -\beta
\end{array}\right],
$$

since the resulting polar coordinate,

$$
\theta(e) \triangleq \arctan \left(\frac{[0,1] W e}{[1,0] W e}\right)
$$

displays the oscillatory dynamics in a particularly simple form,

$$
\dot{\theta}=-\omega \text {. }
$$

Assuming that the augmented dynamics are initially relaxed, $a(0)=(0,0)$, relative motion is guaranteed in the error system as long as the body is not placed at the goal, $(b(0), \dot{b}(0)) \neq 0$. It follows that $\dot{\theta}$ is a nonzero constant, hence, $\theta$ has a zero crossing every $t_{l}=\pi / \omega$ seconds.

\subsection{A Mirror Law for the Augmented Reference Dynamics}

Defining the "mirror strategy"

$$
\begin{array}{cl}
\mu((b, \dot{b}), e)(t) & \triangleq \kappa_{1}(e) b+\kappa_{2}(e) \dot{b} \\
\kappa_{1}(e) & \triangleq\left(k_{1} / \omega\right) \theta(e)+1 \\
\kappa_{2}(e) & \triangleq\left(k_{2} / \omega\right) \theta(e)
\end{array}
$$

now implies that the robot will "overtake" the body, $r(t)=\mu((b, \dot{b}), e)(t)=b(t)$, if and only if

$$
k^{\mathrm{T}}[b, \dot{b}](t)=0 \text { or } \theta(e)(t)=0 .
$$

Now assign the gripper the stratgegy

$$
\operatorname{GRIP}(t)=\left\{\begin{array}{cc}
0: & k^{\mathrm{T}}[b, \dot{b}]=0 \\
1: & k^{\mathrm{T}}[b, \dot{b}] \neq 0
\end{array}\right.
$$

so that the robot cannot engage the body unless $\theta=$ 0 , and this occurs at time $t_{n}=n t_{I}, n=0,1,2 \ldots$. Thus, $\Delta t_{n}$ in (4), is effectively set uniformly to $t_{I}$. At the moment of collision, the robot has the velocity

$$
\dot{r}\left(t_{n}\right)=k_{1} b\left(t_{n}\right)+\left(k_{2}+1\right) \dot{b}\left(t_{n}\right)
$$

and this implies

$$
\begin{aligned}
u(n) & =\dot{r}\left(t_{n}\right)-\dot{b}\left(t_{n}\right) \\
& =k_{1} b\left(t_{n}\right)+k_{2} b\left(t_{n}\right) \\
& =k^{\mathrm{T}} w(n),
\end{aligned}
$$

realizing ${ }^{3}$ any desired linear impact schedule (5).

\footnotetext{
${ }^{3}$ Note that the gripper is disengaged, GRIP $=0$, at time $t_{n}$, if and only if the desired impact schedule called for a zero impulse to be applied anyway.
}

\section{Conclusion}

The mirror law proposed above solves the partial one degree of freedom unit assembly problem. Any (standard) asymptotically exact tracking method that forces the robot to track this $\mu$ results in a trajectory delivering the necessary impact schedule to guarantee that every initial error in actual body state be asymptotically reduced to zero through repeated impacts. It must be emphatically stated that this solution does not address the more fundamental problem of stabilizing a nonholonomically constrained system. The partial problem requires stabilization to a subspace (i.e. bring the body-robot system to the body's goal) to which there are no smoothness obstructions. The full problem (i.e., bring the body-robot system to the body's goal and the robot's nest) remains.

A solution to the full problem should presumably cause the robot to nest if and only if the body is at its goal point. How this should be accomplished is not entirely clear at present. For one would wish to generalize such strategies to encompass both more bodies and higher degrees of freedom. The latter generalization seems reasonably achievable within the present context. The former generalization seems to require a more methodical means of point stabilization in nonholonomically constrained systems.

\section{Acknowledgements}

The author thanks Dr. Ernest Kent for calling his attention to the nonholonomic nature of assembly problems.

\section{References}

[1] Anthony M. Bloch and N. Harris McClamroch. Control of mechanical systems with classical nonholonomic constraints. In Proc. 28th IEEE Conf. on Decision and Control, pages 201-205, Tampa, FL, Dec 1989.

[2] Roger W. Brockett. Asymptotic stability and feedback stabilization. In Roger W. Brockett, Richard S. Millman, and Hector J. Sussman, editors, Differential Geometric Control Theory, chapter 3, pages 181-191. Birkhäuser, 1983.

[3] M. Bühler and D. E. Koditschek. From stable to chaotic juggling. In Proc. IEEE International Conference on Robotics and Automation, pages 1976-1981, Cincinnati, OH, May 1990. 
[4] M. Bühler, D. E. Koditschek, and P. J. Kindlmann. Planning and control of a juggling robot. Int. J. Rob. Research, (submitted), 1991 .

[5] M. Bühler, D. E. Koditschek, and P.J. Kindlmann. A family of robot control strategies for in. termittent dynamical environments. IEEE Control Systems Magazine, 10:16-22, Feb 1990.

[6] Morris W. Hirsch and Stephen Smale. Differential Equations, Dynamical Systems, and Linear Algebra. Academic Press, Inc., Orlando, Fla., 1974.

[7] C. W. Kilmister and J. E. Reeve. Rational Mechanics. Longmans, London, 1966.

[8] Daniel E. Koditschek. Task encoding for autonomous machines: The assembly problem. In Sizth Yale Workshop on Adaptive and Learning Systems, pages 231-236, New Haven, CT, Aug 1990. Center for Systems Science, Yale University.

[9] P. S. Krishnaprasad. Eulerian Many Body Problems. In J. Marsden, Krishnaprasad, and J. Simo, editors, Control Theory and Multibody Systems, volume 97 , pages $187-208$. AMS Series in Contemporary Mathematics, 1989.

[10] Tomás Lozano-Perez, Matthew T. Mason, and Russell H. Taylor. Automatic synthesis of finemotion strategies for robots. The International Journal of Robotics Research, 3(1):3-23, 1984.

[11] R. Murray and S. Sastry. Mathematical problems in grasping and manipulation by multifingered robot hands. In Mathematical Questions in Robotics, Lecture Notes, Providence, RI, 1990. American Mathematical Society.

[12] D. E. Whitney. Force feedback control of manipulator fine motions. ASME J. Dyn. Syst., 98:9197, June 1977. 